lich den irritierenden Umstand, daß weitreichende Thesen en passant und ohne sonderlichen Begründungsaufwand aufgestellt, ihre Konsequenzen nicht diskutiert werden. Auf S. 39 behauptet der Autor schlicht, ,,innere Angelegenheiten“ könnten aus Rechtsgründen nicht Gegenstand zwischenstaatlicher Verhandlungen sein - unrealistischer geht es nicht.

Philip Kunig

RAYMONDE Gour-TANGUAy (ed.)

\title{
Environmental Policies in Developing Countries
}

Beiträge zur Umweltgestaltung, Heft A 27, zusammengestellt von $\mathrm{H}$. und J. M. Johnson, Erich Schmidt Verlag, Berlin, 1977, VIII, 706 S., 48,- DM

Das Buch enthält die Ergebnisse einer 1974 begonnenen Umfrage bei den Regierungen von 102 Staaten zum Stand ihrer nationalen Umweltpolitik und -gesetzgebung. In 63 Fällen erschien den Autoren das Ergebnis einer Veröffentlichung wert. Afrika ist nahezu vollständig vertreten, auch die asiatische (allerdings fehlt die Volksrepublik China) und arabische Welt ist gut repräsentiert. Amerikanische Staaten sind hingegen nur fünf bearbeitet worden (wobei die interessantesten, nämlich Brasilien, Mexiko, Venezuela fehlen). Ergänzt wird das Bild durch Bulgarien, Jugoslawien, Zypern.

Die einzelnen Beiträge sind einheitlich gegliedert und bieten tabellarische Ubersichten mit gelegentlichen kommentierenden Bemerkungen. Nach einer völligen überflüssigen und schon bei Erscheinen des Buches überholten Tabelle politischer, sozialer und ökonomischer Rahmenbedingungen (jeweils knapp eine Seite) finden sich Einträge zur Umweltpolitik im allgemeinen, zum Umweltschutz bei der Nutzung natürlicher Ressourcen, zur Umweltplanung sowie zum Stand der Umweltforschung und -technologie. Knappe Bibliographien, die viel offizielle und offiziöse Dokumente enthalten, schließen jeden Länderbericht ab. Die Abschnitte zur Ressourcennutzung und zur Planung sind übersichtlich nach Sachbereichen gegliedert, jeder Gliederungspunkt zerfällt wiederum in die Abschnitte ,,Problems“, ,,Policy“, ,,Legislation“", , Administration“. Dieses detaillierte Schema wird zu jedem Staat abgedruckt, enthält aber beileibe nicht zu jedem Punkt auch eine Aussage; dies ist bei vielen Berichten geradezu nur vereinzelt der Fall, so daß der Umfang des Buches drastisch hätte reduziert werden können.

Die statt dessen gewählte Vorgehensweise rechtfertigt sich auch nicht dadurch, daß das Fehlen einer Angabe auf das Nichtbestehen eines Problems oder etwa einer gesetzlichen Vorschrift rückschließen ließe, denn häufig wird einfach die schlechte Informationslage für die Lücke ursächlich sein.

Es kann dennoch kein Zweifel sein, daß die Publikation von großem Nutzen ist. In einem fast noch nicht dokumentierten, aber für künftige nationale und internationale Entwicklungen immens wichtigen Feld ermöglicht sie eine erste Information, liefert Material für künftige empirische Einzelstudien und arbeitet damit der noch zu leistenden Aufgabe einer Erforschung des Verhältnisses von Industrialisierung und Umweltschutz in Entwicklungsländern vor. In der in den nächsten Jahren, stimuliert auch durch das Bevorstehen der zweiten Stockholmer Umweltkonferenz der Vereinten Nationen (1982), hierüber verstärkt zu führenden politischen und wissenschaftlichen Diskussionen wird sich das Werk gewiß als nützlich erweisen.

Philip Kunig 